\title{
Signal Representation for Compression and Noise Reduction Through Frame-Based Wavelets
}

\author{
Laura Rebollo-Neira, Anthony G. Constantinides, Senior Member, IEEE, and Tania Stathaki
}

\begin{abstract}
A mathematical framework for data representation and for noise reduction is presented in this paper. The basis of the approach lies in the use of wavelets derived from the general theory of frames to construct a subspace capable of representing the original signal excluding the noise. The representation subspace is shown to be efficient in signal modeling and noise reduction, but it may be accompanied by an ill-conditioned inverse problem. This is further examined, and a more adequate orthonormal representation for the generated subspace is proposed with an improvement in compression performance.
\end{abstract}

Index Terms - Frames, noise suppression, signal compression, wavelets.

\section{INTRODUCTION}

$\mathbf{T}$ WE representation of a signal $f(t)$ as a discrete set of numbers $c_{j}$, which is related through some functional form to $f(t)$, other than the samples $f\left(t_{i}\right)$, is essentially a problem in signal modeling. When the cardinality of $c_{j}$ is less than that of $f\left(t_{i}\right)$, then we have data compression. A classical way to carry out the signal modeling is through the decomposition of $f(t)$ into some functions $\phi_{j}(t)$

$$
f(t)=\sum_{j \in Z} c_{j} \phi_{j}(t)
$$

If the signal space is restricted to the Hilbert space, then to reduce asymptotically the error of representation, the functions $\phi_{j}(t) ; j \in Z$ must form a complete set in the same space. This property guarantees that every finite $L^{2}$-norm function $f(t)$ admits a decomposition of the form (1), which converges strongly to $f(t)$. Furthermore, for the compression to be effective, the functions $\phi_{j}(t) ; j \in Z$ must be chosen such that the number of coefficients $c_{j}$ is significantly less than the number of samples $f\left(t_{i}\right)$ required to retain the desired signal information. Some functions known to be endowed with both properties are the so-called wavelets [5].

Wavelets arise as translations and dilations of a single prototype function. The dilation operator is defined as [5], [7]

$$
\hat{D}_{a} \phi(t)=|a|^{-(1 / 2)} \phi\left(\frac{t}{a}\right)
$$

and the translation operator as

$$
\hat{T}_{b} \phi(t)=\phi(t-b) .
$$

Manuscript received January 31, 1995; revised February 18, 1997. The associate editor coordinating the review of this paper and approving it for publication was Dr. Farokh Marvasti.

The authors are with the Department of Electrical and Electronic Engineering, Imperial College, London, U.K.

Publisher Item Identifier S 1053-587X(98)01995-3.
If the parameter $a$ takes discrete values $a_{0}^{m}$ and the parameter $b$ takes discrete values $n b_{0}$, then a discrete set of wavelets $\phi_{m, n}(t) ; m, n \in Z$ is obtained from a "mother" function $\phi(t)$ through the operations

$$
\phi_{m, n}(t)=\hat{D}_{a_{0}^{m}} \hat{T}_{n b_{0}} \phi(t)=\left|a_{0}\right|^{-(m / 2)} \phi\left(a_{0}^{-m} t-n b_{0}\right) .
$$

The fundamental property the mother function $\phi(t)$ [as well as its Fourier transform $\breve{\phi}(\omega)$ ] must have to render the set $\phi_{m, n}(t) ; m, n \in Z$ useful for compression purposes is that of fast decay in the time and in the frequency domains [4], [5].

The decomposition of a signal in terms of wavelets adopts the form

$$
f(t)=\sum_{n \in Z} \sum_{m \in Z} c_{m, n} \phi_{m, n}(t)
$$

While orthogonality is not essential, when the set $\phi_{m, n}(t)$; $m, n \in Z$ is an orthonormal set, the coefficients $c_{m, n}$ are calculated in a straightforward manner as the inner products $\left\langle\phi_{m, n} \mid f\right\rangle$. Although orthogonal wavelets with reasonable fast decay in both the time and frequency domains have been constructed [3], [8], [10], the relaxation of the requirements on orthogonality and linear independence can produce wavelets with better localization properties [2], [4]. This less stringent requirement for the construction of wavelets places the concept of signal representation within the mathematical theory of "frames" [6].

Basically, a frame is a complete set of functions and is not necessarily a basis. In [2], [4], and [5], the conditions under which the functions $\phi_{m, n}(t) ; m, n \in Z$ constitute a frame are extensively studied. The frame condition is imposed not only to guarantee completeness of the set but also to ensure that the mapping (5) has a bounded inverse. In addition to a good compression performance, the aim of the present work is to reduce simultaneously the influence of random noise on the original signal. To this end, we propose the use of "well located" wavelets to define a "working" subspace capable of representing the uncontaminated signal and incapable to reproduce noise.

Even though we restrict our considerations to frame-based wavelets only (henceforth referred to as frame wavelets), the fact that we deal with a finite subset of frame elements, and that we build the dual vectors in this subspace, implies that no inverse mapping is required to exist. This is a deviation from normal frame analysis, where such an inverse mapping is considered mandatory. For the problem at hand, this is not an inconvenience as the objective of the problem also allows us to restrict the space of solutions to that in which the inverse does 
exist and is a minimum norm solution. However, difficulties of more fundamental nature appear in connection with situations in which the spectrum of the kernel $\left\langle\phi_{m, n} \mid \phi_{l, k}\right\rangle$ has a fast decay rate. For kernels having this property, the inversion becomes an ill-conditioned problem whereby the norm of the coefficients, which are used to represent the signal, yields a large numerical answer. It is important to remark that this problem arises when one constructs the dual vectors in a subspace spanned by a finite number of frame elements. If the dual frame in the Hilbert space were used to calculate the coefficients of an expansion in terms of the same frame elements, although not all signals belonging to such a subspace can be expressed in terms of coefficients so calculated, the norm of these coefficients is guaranteed to be finite. We analyze here the causes of the misbehavior inherent in building the dual vectors in the working subspace. Our treatment shows clearly that the problem can be avoided simply by adopting an orthonormal representation for the wavelet-generated subspace. From the mathematical approach we present, a method to construct explicitly the orthonormal representation evolves naturally. The new representation preserves the signal norm and improves compactness of the subspace with respect to its compression properties.

The paper is organized as follows. In Section II, the notation to be used is introduced. In Section III, the frame definition is given, and the working subspace referred to above is constructed. Section IV deals with the inverse problem, and an orthonormal representation is proposed. In Section V, the approach is illustrated by using the wavelets given in [5]. Numerical tests are performed in Section VI, where a procedure to reduce random noise is considered. Finally, conclusions are drawn in Section VII.

\section{NOTATION}

We assume that the possible signals to be considered are elements of a Hilbert space, and we adopt the Dirac notation [1] for such elements. In this notation, a vector $f$ is represented as $|f\rangle$ and its dual as $\langle f|$. Given a complete set of discrete orthonormal vectors $\left\{|j\rangle ;\langle k \mid j\rangle=\delta_{k, j} ; k, j \in Z\right\}$ or a set of $\delta$-normalized continuous orthonormal vectors $\left\{|t\rangle ;\left\langle t^{\prime} \mid t\right\rangle=\right.$ $\left.\delta\left(t-t^{\prime}\right) ; t, t^{\prime} \in R\right\}$, the resolution of unity is expressed as the operators

$$
\begin{aligned}
& \hat{I}=\sum_{j \in Z}|j\rangle\langle j| \\
& \hat{I}=\int|t\rangle\langle t| d t .
\end{aligned}
$$

Through these operators, two functional representations of a Hilbert space can be introduced. Simply by inserting the unit operator as in

$$
\langle g \mid f\rangle=\sum_{j \in Z}\langle g \mid j\rangle\langle j \mid f\rangle
$$

and

$$
\langle g \mid f\rangle=\int\langle g \mid t\rangle\langle t \mid f\rangle d t
$$

we are automatically led to two representations of the Hilbert space. Equation (8) gives a representation by $l^{2}(Z)$, which is the space of square summable sequences, with $f_{j}=\langle j \mid f\rangle$ and $\overline{g_{j}}=\langle g \mid j\rangle=\overline{\langle j \mid g\rangle}$, where $\overline{g_{j}}$ denotes the complex conjugate of $g_{j}$, whereas (9) leads to a representation by $H=L^{2}(R)$, which is the space of square integrable functions, with $f(t)=\langle t \mid f\rangle$ and $\overline{g(t)}=\langle g \mid t\rangle=\overline{\langle t \mid g\rangle}$.

As usual, $\||f\rangle \|=\langle f \mid f\rangle^{1 / 2}$ defines the vector norm, and the norm of a operator $\hat{A}$ is defined as

$$
\|\hat{A}\|=\sup _{\||f\rangle \|=1} \| \hat{A}|f\rangle \| .
$$

A Hermitian operator having a discrete set of eigenvectors $\left|\psi_{n}\right\rangle$ can be represented through its spectrum as

$$
\hat{A}=\sum_{n \in N}\left|\psi_{n}\right\rangle \lambda_{n}\left\langle\psi_{n}\right|
$$

where $\lambda_{n} ; n \in N$ are its eigenvalues satisfying

$$
\hat{A}\left|\psi_{n}\right\rangle=\lambda_{n}\left|\psi_{n}\right\rangle
$$

with $\left\langle\psi_{m} \mid \psi_{n}\right\rangle=\delta_{m, n}$. If $\lambda_{n} \neq 0 ; n \in N$, then $\hat{A}$ is invertible, and the spectral decomposition of its inverse $\hat{A}^{-1}$ is

$$
\hat{A}^{-1}=\sum_{n \in N}\left|\psi_{n}\right\rangle \frac{1}{\lambda_{n}}\left\langle\psi_{n}\right| .
$$

\section{FRAMES}

The theory of frames was introduced by Duffin and Shaffer [6]. In another context [2], [4], [5], this theoretical framework has been adopted to construct several wavelet functions with good localization properties, in both time and frequency, under the requirement that they constitute a frame.

Definition 1: A set of vectors $\left|\phi_{j}\right\rangle ; j \in Z$ in a Hilbert space $H$ is called a frame if there exist numbers $A, B>0$ such that for all vectors $|f\rangle \in H$

$$
A\langle f \mid f\rangle \leq \sum_{j \in Z}\left|\left\langle\phi_{j} \mid f\right\rangle\right|^{2} \leq B\langle f \mid f\rangle .
$$

The numbers $A, B$ are called the frame bounds [6], [13].

Although a frame need not be a set of independent vectors, it is clear that these vectors constitute a complete set of vectors since the relations $\left\langle\phi_{j} \mid f\right\rangle=0 ; j \in Z$ imply $|f\rangle \equiv 0$. Therefore, any vector $|f\rangle \in H$ can be spanned as

$$
|f\rangle=\sum_{j \in Z}\langle j \mid c\rangle\left|\phi_{j}\right\rangle .
$$

Definition 2: If $\left|\phi_{j}\right\rangle ; j \in Z$ is a frame in $H$, then the frame operator $\hat{F}$ is the linear operator from $H$ to $l^{2}(Z)$ defined by

$$
\langle j|\hat{F}| f\rangle=\left\langle\phi_{j} \mid f\right\rangle .
$$

It follows from (14) that $\hat{F}$ is bounded and, therefore, so is its adjoint $\hat{F}^{*}: l^{2}(Z) \rightarrow H$. According to its definition, the frame operator can be written as

$$
\hat{F}=\sum_{j \in Z}|j\rangle\left\langle\phi_{j}\right|
$$

and the adjoint $\hat{F}^{*}$ as

$$
\hat{F}^{*}=\sum_{j \in Z}\left|\phi_{j}\right\rangle\langle j| .
$$


In terms of $\hat{F}^{*},(15)$ can be recast in the form

$$
|f\rangle=\hat{F}^{*}|c\rangle
$$

and the frame condition (14) as

$$
A \hat{I} \leq \hat{F}^{*} \hat{F} \leq B \hat{I} \text {. }
$$

The proofs of the following propositions are given in [5]:

i) $\hat{F}^{*} \hat{F}$ is invertible, and $B^{-1} \hat{I} \leq\left(\hat{F}^{*} \hat{F}\right)^{-1} \leq A^{-1} \hat{I}$.

ii) The set of vectors $\left\langle\tilde{\phi}_{j}^{H}\right|=\left\langle\phi_{j}\right|\left(\hat{F}^{*} \hat{F}\right)^{-1} ; j \in Z$ is a frame with bounds $B^{-1}, A^{-1}$ called the dual frame of $\left|\phi_{j}\right\rangle ; j \in Z$

iii) Every $|f\rangle \in H$ can be written as

$$
|f\rangle=\sum_{j \in Z}\left\langle\tilde{\phi}_{j}^{H} \mid f\right\rangle\left|\phi_{j}\right\rangle
$$

These propositions are a consequence of the frame condition (20), which ensures that the operator $\hat{F}^{*} \hat{F}$ has an inverse. In this paper, we address the problem that arises when one intends to obtain the dual vectors in a subspace spanned by a finite number of frame elements in $H$.

Let $Z_{J} \subset Z$ be a subset of $J$ elements of $Z$, and let $S$ be the subspace generated by the vectors $\left|\phi_{j}\right\rangle ; j \in Z_{J}$. If the subspace is constructed in this arbitrary manner then, although for all $|f\rangle \in S$ the upper bound in (14) will be satisfied, the lower bound may become too small to be useful in practice. In such cases, the operator (19) has no inverse in a strict sense. However, as is discussed below, by restricting the domain of transformation (19) to a particular subspace of $l^{2}\left(Z_{J}\right)$ the inversion of such a map is rendered possible, but it may result in an ill-conditioned problem. In the next section, we discuss the problem of solving (19) for those cases in which the operator $\hat{F}^{*}$ is not bounded from below, and we show that the same approach also provides an appropriate orthonormal representation.

\section{The InVersion Problem AND A More ECONOMICAL ORTHONORMAL REPRESENTATION}

Initially, we construct the operator $\hat{F}_{J}$ from $S$ to $l^{2}\left(Z_{J}\right)$ as

$$
\hat{F}_{J}=\sum_{j \in Z_{J}}|j\rangle\left\langle\phi_{j}\right| .
$$

Then, its adjoint $\hat{F}_{J}^{*}$ from $l^{2}\left(Z_{J}\right)$ to $S$ is given by

$$
\hat{F}_{J}^{*}=\sum_{j \in Z_{J}}\left|\phi_{j}\right\rangle\langle j|
$$

so that for any vector $|f\rangle \in S$, we have

$$
|f\rangle=\hat{F}_{J}^{*}|c\rangle
$$

for some $|c\rangle \in l^{2}\left(Z_{J}\right)$.

In order to enable the inversion of the mapping (24), we look for the eigenvectors of the operator $\hat{F}_{J} \hat{F}_{J}^{*}: l^{2}\left(Z_{J}\right) \rightarrow l^{2}\left(Z_{J}\right)$. This is a bounded operator, as $\hat{F}_{J}$ and $\hat{F}_{J}^{*}$ are from hypothesis bounded, and which is also self-adjoint. Therefore, from the
Hilbert-Schmidt theorem [11], $\hat{F}_{J} \hat{F}_{J}^{*}$ has a complete set of eigenvectors that satisfy the conditions

$$
\begin{gathered}
\hat{F}_{J} \hat{F}_{J}^{*}\left|\psi_{n}\right\rangle=\lambda_{n}\left|\psi_{n}\right\rangle ; \quad\left\langle\psi_{k} \mid \psi_{n}\right\rangle=\delta_{k, n} \\
n=1, \cdots, J
\end{gathered}
$$

with eigenvalues $\lambda_{n} \geq 0 ; n=1, \cdots, J$.

Since this set of orthonormal vectors is complete, it provides a representation for the orthogonal projection operator onto $l^{2}\left(Z_{J}\right)$. On the other hand, the set of vectors $\left|\varphi_{n}\right\rangle \in S$ obtained through the map $\left|\varphi_{n}\right\rangle=\hat{F}_{J}^{*}\left|\psi_{n}\right\rangle$ is also orthogonal since $\left\langle\varphi_{n} \mid \varphi_{k}\right\rangle=\left\langle\psi_{n}\left|\hat{F}_{J} \hat{F}_{J}^{*}\right| \psi_{k}\right\rangle=\lambda_{k} \delta_{n, k}$, which also implies that the eigenvectors $\left|\psi_{n}\right\rangle$ corresponding to zero eigenvalues give rise to vectors $\left|\varphi_{n}\right\rangle$ of zero norm. It is clear then that the eigenvectors $\left|\psi_{n}\right\rangle$ corresponding to zero eigenvalues span the space $\operatorname{Nul}\left(\hat{F}_{J}^{*}\right)=\left\{|c\rangle \in l^{2}\left(Z_{J}\right) ; \hat{F}_{J}^{*}|c\rangle=\right.$ $0\}$ and the vectors $\left|\varphi_{n}\right\rangle$ for $\lambda_{n} \neq 0$ span $\operatorname{Ran}\left(\hat{F}_{J}^{*}\right)=\{|f\rangle \in S$; $|f\rangle=\hat{F}_{J}^{*}|c\rangle$; for some $\left.|c\rangle \in l^{2}\left(Z_{J}\right)\right\}$. This fact means that it is sufficient to restrict $\hat{F}_{J}^{*}$ to act only on $\operatorname{Nul}\left(\hat{F}_{J}^{*}\right)^{\perp}$, which is the orthogonal complement of $\operatorname{Nul}\left(\hat{F}_{J}^{*}\right)$ so that the inversion of such a map can be made possible.

Let $\hat{F}_{J}^{* \prime}$ be the restriction of $\hat{F}_{J}^{*}$ to $\operatorname{Nul}\left(\hat{F}_{J}^{*}\right)^{\perp}$. The normalized vectors

$$
\left|\bar{\varphi}_{n}\right\rangle=\frac{\left|\varphi_{n}\right\rangle}{\lambda_{n}^{1 / 2}}=\frac{\hat{F}_{J}^{* \prime}\left|\psi_{n}\right\rangle}{\lambda_{n}^{1 / 2}}
$$

with $\lambda_{n} \neq 0$ are the eigenvectors of the operator $\hat{F}_{J}^{* \prime} \hat{F}_{J}$ with corresponding eigenvalues $\lambda_{n}$, as readily follows. The spectral decomposition of $\hat{F}_{J}^{* \prime} \hat{F}_{J}$ is then

$$
\hat{F}_{J}^{* \prime} \hat{F}_{J}=\sum_{n=1, \lambda_{n} \neq 0}^{J}\left|\bar{\varphi}_{n}\right\rangle \lambda_{n}\left\langle\bar{\varphi}_{n}\right|
$$

and the corresponding decomposition for its inverse is

$$
\left(\hat{F}_{J}^{* \prime} \hat{F}_{J}\right)^{-1}=\sum_{n=1, \lambda_{n} \neq 0}^{J}\left|\bar{\varphi}_{n}\right\rangle \frac{1}{\lambda_{n}}\left\langle\bar{\varphi}_{n}\right| .
$$

Moreover

$$
\hat{F}_{J}^{* \prime}=\sum_{n=1, \lambda_{n} \neq 0}^{J}\left|\bar{\varphi}_{n}\right\rangle \lambda_{n}^{1 / 2}\left\langle\psi_{n}\right|
$$

since (29) satisfies (26). The numbers $\beta_{n}=\lambda_{n}^{1 / 2}$ are called the singular values of $\hat{F}_{J}^{*}$ and $\left|\bar{\varphi}_{n}\right\rangle$ and $\left|\psi_{n}\right\rangle$ its left and right singular vectors, respectively. The inverse operator $\hat{F}_{J}^{* \prime-1}$ from $\operatorname{Ran}\left(\hat{F}_{J}^{*}\right)$ to $\operatorname{Nul}\left(\hat{F}_{J}^{*}\right)^{\perp}$ results in

$$
\hat{F}_{J}^{* \prime-1}=\sum_{n=1, \lambda_{n} \neq 0}^{J}\left|\psi_{n}\right\rangle \frac{1}{\beta_{n}}\left\langle\bar{\varphi}_{n}\right| .
$$

Both (28) and (30) render the representation of a vector $|f\rangle \in S$ in terms of the set $\left|\phi_{j}\right\rangle ; j \in Z_{J}$ since $\hat{F}_{J}^{* \prime-1}=\hat{F}_{J}\left(\hat{F}_{J}^{* \prime} \hat{F}_{J}\right)^{-1}$, and then, $\langle j \mid c\rangle=\left\langle j\left|\hat{F}_{J}^{* \prime-1}\right| f\right\rangle=$ $\left\langle j\left|\hat{F}_{J}\left(\hat{F}_{J}^{* \prime} \hat{F}_{J}\right)^{-1}\right| f\right\rangle$. 
The vectors

$$
\begin{aligned}
\left\langle\tilde{\phi}_{j}\right| & =\langle j| \hat{F}_{J}^{* \prime-1}=\langle j| \hat{F}_{J}\left(\hat{F}_{J}^{* \prime} \hat{F}_{J}\right)^{-1}=\left\langle\phi_{j}\right|\left(\hat{F}_{J}^{* \prime} \hat{F}_{J}\right)^{-1} \\
& =\sum_{n=1, \lambda_{n} \neq 0}^{J}\left\langle j \mid \psi_{n}\right\rangle \frac{1}{\beta_{n}}\left\langle\bar{\varphi}_{n}\right| ; \quad j \in Z_{J}
\end{aligned}
$$

produce a representation of $|f\rangle$ as given by

$$
|f\rangle=\sum_{j \in Z_{J}}\langle j \mid c\rangle\left|\phi_{j}\right\rangle=\sum_{j \in Z_{J}}\left\langle\widetilde{\phi}_{j} \mid f\right\rangle\left|\phi_{j}\right\rangle
$$

which looks identical to the expansion of $|f\rangle$ into biorthogonal bases. However, when an eigenvalue is zero, then the set $\left|\phi_{j}\right\rangle ; j \in Z_{J}$ does not constitute a basis, and therefore, the set $\left\langle\tilde{\phi}_{j}\right| ; j \in Z_{J}$ is not a biorthogonal set. This appears clearly when the inner products $\left\langle\tilde{\phi}_{j} \mid \phi_{k}\right\rangle$ are calculated as

$$
\left\langle\tilde{\phi}_{j} \mid \phi_{k}\right\rangle=\sum_{n=1, \lambda_{n} \neq 0}^{J}\left\langle j \mid \psi_{n}\right\rangle\left\langle\psi_{n} \mid k\right\rangle
$$

The right-hand side of (33) is equal to $\delta_{j, k}$ only if the sum runs over all $Z_{J}$. Since, in (33), the sum is restricted to $\lambda_{n} \neq 0$, it follows that $\left\langle\tilde{\phi}_{j} \mid \phi_{k}\right\rangle \neq \delta_{j, k}$ when $\operatorname{Nul}\left(\hat{F}_{J}^{*}\right) \neq\{\emptyset\}$.

The existence of zero eigenvalues also implies that the representation (32) is not unique since if $\hat{F}_{J}^{*}|c\rangle=|f\rangle$, then any other vector $|b\rangle=|c\rangle+\left|c^{\circ}\right\rangle$ with $\left|c^{\circ}\right\rangle \in \operatorname{Nul}\left(\hat{F}_{J}^{*}\right)$ and $|c\rangle \in \operatorname{Nul}\left(\hat{F}_{J}^{*}\right)^{\perp}$ will give the same representation of $|f\rangle$ since $\hat{F}_{J}^{*}\left|c^{\circ}\right\rangle=0$. Thus, by choosing $\left|c^{\circ}\right\rangle=0$, the solution of minimum norm may be obtained. As a solution of this type is suitable for the problem we are addressing here, the lack of uniqueness in the general case is not a difficulty. However, it may happen that when the spectrum of the operator $\hat{F}_{J} \hat{F}_{J}^{* \prime}$ has a fast decay rate, then the representation (32) becomes "noneconomical." This is evident from the computation of $\left\langle\tilde{\phi}_{j} \mid f\right\rangle$ in which the eigenvalues appear in the denominator

$$
\left\langle\widetilde{\phi}_{j} \mid f\right\rangle=\sum_{n=1}^{r}\left\langle j \mid \psi_{n}\right\rangle \frac{1}{\lambda_{n}^{1 / 2}}\left\langle\bar{\varphi}_{n} \mid f\right\rangle ; \quad j \in Z_{J}
$$

where the vectors $\left|\psi_{n}\right\rangle$ are now ordered so that $\lambda_{n} \neq 0$ for $1 \leq n \leq r$ and $\lambda_{n}=0$ for $r+1 \leq n \leq J$. If we define as "economical" any representation of $|f\rangle \in S$ and $\||f\rangle \|=1$ such that for some set $\left|\alpha_{j}\right\rangle ; j \in Z_{J}$

$$
|f\rangle=\sum_{j \in Z_{J}} b_{j}\left|\alpha_{j}\right\rangle
$$

and

$$
\sum_{j \in Z_{J}}\left|b_{j}\right|^{2} \leq E
$$

with $E \geq 1$, which is a given number that defines "economy," it is seen that due to the existence of small eigenvalues, the representation (32) may not satisfy (36). In fact, it follows from (34) that

$$
\sum_{j \in Z_{J}}\left|\left\langle\widetilde{\phi}_{j} \mid f\right\rangle\right|^{2}=\sum_{n=1}^{r} \frac{\left|\left\langle\bar{\varphi}_{n} \mid f\right\rangle\right|^{2}}{\lambda_{n}}
$$

and hence, the small eigenvalues force (37) to result in a very large number.
This problem can be overcome without additional work simply by adopting the orthonormal representation for the subspace $S$, which is provided by the set of vectors $\left|\bar{\varphi}_{n}\right\rangle ; n=$ $1, \cdots, r$. As already discussed above, this is an orthonormal set of vectors spanning $\operatorname{Ran}\left(\hat{F}_{J}^{*}\right)$, and therefore, the orthogonal projection onto $\operatorname{Ran}\left(\hat{F}_{J}^{*}\right)$ can be written as

$$
\hat{P}_{R}=\sum_{n=1}^{r}\left|\bar{\varphi}_{n}\right\rangle\left\langle\bar{\varphi}_{n}\right|
$$

and for all $|f\rangle \in S$, we have

$$
|f\rangle=\sum_{n=1}^{r}\left\langle\bar{\varphi}_{n} \mid f\right\rangle\left|\bar{\varphi}_{n}\right\rangle .
$$

The latter is a unitary transformation so that it conserves the norm of $|f\rangle$. In effect, as $\left\langle\bar{\varphi}_{n} \mid \bar{\varphi}_{m}\right\rangle=\delta_{n, m}$, then $\left.\| f\right\rangle \|=$ $1 \Longrightarrow \sum_{n=1}^{r}\left|\left\langle\bar{\varphi}_{n} \mid f\right\rangle\right|^{2}=1$, and hence, the orthonormal representation (39) always satisfies (36). On the contrary, the representation (32) in terms of vectors $\left|\phi_{j}\right\rangle ; j \in Z_{J}$ may not satisfy (36), no matter how reasonably large the "economy" number $E$ is chosen.

Thus far, we have assumed that the signal $|f\rangle$ belongs to $S$, which is the subspace generated through the frame elements $\left|\phi_{j}\right\rangle ; j \in Z_{J}$. If $|f\rangle \notin S$ and $|f\rangle$ is approximated by $|\tilde{f}\rangle$ such that

$$
|\tilde{f}\rangle=\sum_{j \in Z_{J}}\left\langle\tilde{\phi}_{j} \mid f\right\rangle\left|\phi_{j}\right\rangle
$$

or $\left|f^{*}\right\rangle$ such that

$$
\left|f^{*}\right\rangle=\sum_{n=1}^{r}\left\langle\bar{\varphi}_{n} \mid f\right\rangle\left|\bar{\varphi}_{n}\right\rangle
$$

both (40) and (41) yield the same approximation for $|f\rangle$ and is the approximation in $S$ that minimizes the distance to $|f\rangle$. In fact, for $|f\rangle \notin S,|f\rangle-\left|f^{*}\right\rangle \in S^{\perp}$, and then, we have $\left\langle\tilde{\phi}_{j} \mid f\right\rangle=\left\langle\tilde{\phi}_{j} \mid f^{*}\right\rangle$ for all $j \in Z_{J}$; therefore

$$
|\tilde{f}\rangle=\sum_{j \in Z_{J}}\left\langle\tilde{\phi}_{j} \mid f\right\rangle\left|\phi_{j}\right\rangle=\sum_{j \in Z_{J}}\left\langle\tilde{\phi}_{j} \mid f^{*}\right\rangle\left|\phi_{j}\right\rangle=\left|f^{*}\right\rangle
$$

by (32) applied to $\left|f^{*}\right\rangle$. Moreover, if we take an arbitrary vector $|g\rangle \in S$ since $\left\langle f^{*}-f \mid g-f^{*}\right\rangle=0$, we have $\langle g-f \mid g-f\rangle=\left\langle g-f^{*}+f^{*}-f \mid g-f^{*}+f^{*}-f\right\rangle=$ $\left\langle g-f^{*} \mid g-f^{*}\right\rangle+\left\langle f^{*}-f \mid f^{*}-f\right\rangle$; therefore, the distance $\||g-f\rangle||$ is minimized if $|g\rangle \equiv\left|f^{*}\right\rangle$.

We want to stress that if the dual frames in the Hilbert space $\left\langle\tilde{\phi}_{j}^{H}\right|$ are used to build a different approximation $\left|f^{H}\right\rangle$ as

$$
\left|f^{H}\right\rangle=\sum_{j \in Z_{J}}\left\langle\tilde{\phi}_{j}^{H} \mid f\right\rangle\left|\phi_{j}\right\rangle
$$

then when $\||f\rangle||=1$, the norm $\sum_{j \in Z_{J}}\left|\left\langle\tilde{\phi}_{j}^{H} \mid f\right\rangle\right|^{2}$ is bounded by the number $A^{-1}$; however, the approximation (43) does not minimize the distance to $|f\rangle$. 


\section{WAVELET SUBSPACE}

At this point, we fix the subspace $S$ by choosing a finite set of wavelet functions as those proposed in [5]. We restrict the domain of the possible square integrable functions to the interval $[0, T]$. Since, in a decomposition of the type (5), the coefficients are characterized by two indices, the subspace of the square integrable sequences to be considered are $l^{2}\left(Z_{M} \otimes Z_{N}\right)$, where $Z_{M} \subset Z$ and $Z_{N} \subset Z$ are defined as

$$
\begin{aligned}
& Z_{M}=\left\{m \in Z ; m_{1} \leq m \leq m_{2} ; M=m_{2}+1-m_{1}\right\} \\
& Z_{N}=\left\{n \in Z ; n_{1} \leq n \leq n_{2} ; N=n_{2}+1-n_{1}\right\} .
\end{aligned}
$$

The identity operator in such a subspace is then written in the form

$$
\hat{I}_{M N}=\sum_{m \in Z_{M}} \sum_{n \in Z_{N}}|m, n\rangle\langle m, n|
$$

with

$$
\langle m, n \mid k, j\rangle=\delta_{m, k} \delta_{n, j} ; \quad m, k \in Z_{M} ; n, j \in Z_{N} .
$$

The frame elements to be used are proposed in [5, p. 79] as

$$
\left\langle t \mid \phi_{m, n}\right\rangle=\phi_{m, n}(t)=a_{0}^{-(m / 2)} \phi\left(a_{0}^{-m} t-n b_{0}\right)
$$

where

$$
\phi(t)=C\left[-\frac{1}{2} \gamma(t+1)+\gamma(t)-\frac{1}{2} \gamma(t-1)\right] .
$$

$C$ is chosen as $C=6 \sqrt{\frac{70}{1313}}$ to make $\||\phi\rangle \|=1$, and

$$
\gamma(t)= \begin{cases}\frac{1}{6}(t+2)^{3} ; & -2 \leq t \leq-1 \\ \frac{2}{3}-t^{2}\left(1+\frac{t}{2}\right) ; & -1 \leq t \leq 0 \\ \frac{2}{3}-t^{2}\left(1-\frac{t}{2}\right) ; & 0 \leq t \leq 1 \\ -\frac{1}{6}(t-2)^{3} ; & 1 \leq t \leq 2 \\ 0 & \text { otherwise. }\end{cases}
$$

where

constitute a frame in $L^{2}(R)$ with frame bounds $A=233854$ and $B=2.66717$ for $m, n \in Z$ [5]. Since the infinite set is truncated to a finite one and since we are working in $L^{2}([0, T])$, the lower bound $A$ has lost its relevance. Indeed, in the inversion process we have presented earlier, it plays no role. However, it is appropriate to stress the importance of the fact that the infinite family $\left|\phi_{m, n}\right\rangle ; m, n \in Z$ constitutes a frame in $L^{2}(R)$. As a frame is complete, we can be certain that by increasing $Z_{M}$ and $Z_{N}$, we can obtain a representation for any function in $L^{2}([0, T])$ within the desired degree of approximation.

In order to gain accuracy in the determination of the eigenvalues to be considered as different from zero, we calculate directly the singular values and singular vectors of the operator $\hat{F}_{M N}^{*}$. The numerical representation of this operator is evaluated by the matrix elements

$$
\begin{aligned}
\left\langle t_{i}\left|\hat{F}_{M N}^{*}\right| k, j\right\rangle & =\sum_{m \in Z_{M}} \sum_{n \in Z_{N}}\left\langle t_{i} \mid \phi_{m, n}\right\rangle\langle m, n \mid k, j\rangle \\
= & \phi_{k, j}\left(t_{i}\right) ; \quad i=1, \cdots, N_{T} ; k \in Z_{M} \\
j \in Z_{N} &
\end{aligned}
$$

$$
\left\langle\tilde{\phi}_{m, n} \mid f\right\rangle=\sum_{k \in Z_{M}} \sum_{j \in Z_{N}} \sum_{l=1}^{r}\left\langle m, n \mid \psi_{l}\right\rangle \frac{1}{\lambda_{l}}\left\langle\psi_{l} \mid k, j\right\rangle\left\langle\phi_{k, j} \mid f\right\rangle
$$

and

$$
\left|f^{*}\right\rangle=\sum_{l=1}^{r}\left\langle\bar{\varphi}_{l} \mid f\right\rangle\left|\overline{\varphi_{l}}\right\rangle
$$

where $N_{T}$ is the number of samples that are taken in discretizing the $[0, T]$ interval.

To calculate the singular values $\beta_{n}$ of this matrix, we have used the double precision routine f02wcf of the NAG FORTRAN library [9] (see [12] for full details on the algorithms

$$
\beta_{1} \geq \beta_{2} \geq \cdots \geq \beta_{r} \geq \beta_{r+1} \geq \cdots \geq \beta_{J}
$$

according to the algorithm that is used to calculate the singular values, those to be considered as zero are the ones that satisfy the relation [9], [12]

$$
\beta_{r}<\beta_{1} \mathrm{TOL}
$$

where TOL is a number that should be chosen as approximately the largest relative error in the matrix elements. However, as we discuss below, when the spectrum $\beta_{r}$ does not have a clear cut, the singular values to be disregarded, in order to "regularize" the representation in terms of wavelets, can be set by looking at the orthogonal representation and setting to zero all the singular values corresponding to coefficients of

The two identical approximations of $|f\rangle$ in the subspace $S$, $|\tilde{f}\rangle$, and $\left|f^{*}\right\rangle$ are evaluated as

$$
|\tilde{f}\rangle=\sum_{m \in Z_{M}} \sum_{n \in Z_{N}}\left\langle\tilde{\phi}_{m, n} \mid f\right\rangle\left|\phi_{m, n}\right\rangle
$$

where

$$
\left\langle\bar{\varphi}_{l} \mid f\right\rangle=\frac{1}{\beta_{l}} \sum_{k \in Z_{M}} \sum_{j \in Z_{N}}\left\langle\psi_{l} \mid k, j\right\rangle\left\langle\phi_{k, j} \mid f\right\rangle .
$$

There is still the question concerning the problem of determining the subsets $Z_{M}$ and $Z_{N}$. Since we address this problem by taking into account the errors in the data, this question is the subject of the next section, where simulations are performed.

\section{The Subsets $Z_{M}, Z_{N}$, AND Numerical Tests}

In all the examples given here, the data are noisy signals simulated as

$$
\begin{gathered}
\left\langle t_{i} \mid f\right\rangle=\left\langle t_{i} \mid f_{c}\right\rangle+\left\langle t_{i} \mid \varepsilon\right\rangle ; \quad t_{i}=(i-1) \frac{T}{N_{T}} \\
i=1, \cdots, N_{T}
\end{gathered}
$$

where $\left\langle t_{i} \mid f_{c}\right\rangle$ are the sampling values of the clean signal, and $\left\langle t_{i} \mid \varepsilon\right\rangle$ are random Gaussian identically distributed variables 
with mean zero and variance $\sigma^{2}$. From the sampling data, the inner products in (54) and (56) are calculated as

$$
\left\langle\phi_{m, n} \mid f\right\rangle=\sum_{i=1}^{N_{T}}\left\langle\phi_{m, n} \mid t_{i}\right\rangle\left\langle t_{i} \mid f\right\rangle
$$

where the right-hand side acts only as an approximation to the left-hand side in the form of a Riemman sum. In Fig. 1(a), the mother wavelet $\phi(t)$ is plotted, and Fig. 1(b) and (c) shows $\phi_{-2,3}(t)$ and $\phi_{-3,3}(t)$, respectively. These figures exemplify the fact that the functions $\phi_{m, n}(t)$ become sharper as $m$ decreases. This feature renders the functions for large negative value of $m$ susceptible to reproducing random noise, and hence, in order to make the contribution of noise insignificant, i.e., to leave the noise outside the subspace $S$, we must set $m_{1}$, in $Z_{M}$, as large as possible. The upper bound $m_{2}$ need not be precise, and it can be overestimated without causing undue effects. The bounds $n_{1}$ and $n_{2}$ for $Z_{N}$ are merely estimated so that the interval $[0, T]$ is covered by the support of the functions involved. Thus, the crucial choice is with respect to $m_{1}$. In order to fix its value, it is necessary to specify the degree of approximation to which the input signal has to be reproduced. An appropriate criterion should take into account the imprecision associated with the data. We propose, therefore, to fix $m_{1}$ as the maximum value for which

$$
\boldsymbol{E}\left\{|||\tilde{f}\rangle-|f\rangle \|^{2}\right\}=\boldsymbol{E}\left\{||\left|f^{*}\right\rangle-|f\rangle \|^{2}\right\} \leq \sigma^{2}
$$

is satisfied. $\boldsymbol{E}\{$.$\} denotes the expectation operator, and for$ practical purposes, it may be taken as the mean value operation.

In the absence of noise, $\sigma=0$ and (59) is reduced to $\left|f^{*}\right\rangle \equiv|\tilde{f}\rangle \equiv|f\rangle$. The discrete version of (59) is given by

$$
\frac{1}{N_{T}} \sum_{i=1}^{N_{T}}\left(\left\langle t_{i} \mid f\right\rangle-\left\langle t_{i} \mid \tilde{f}\right\rangle\right)^{2}=\frac{1}{N_{T}} \sum_{i=1}^{N_{T}}\left(\left\langle t_{i} \mid f\right\rangle-\left\langle t_{i} \mid f^{*}\right\rangle\right)^{2} \leq \sigma^{2} .
$$

In the simulations below, we start initially with $m_{1}=-1$, i.e., we set as initial value the first negative value of $m_{1}$ from which point a few iterations are needed to determine the larger value of $m_{1}$ such that (60) is satisfied.

First, we present two standard signals: a truncated ramp with discontinuities on each side and a chirp pulse. In both cases, $T=1$ and the standard deviation of the noise is fixed as $20 \%$ of the maximum value reached by the clean signal. This corresponds to $\sigma=0.15$ for the ramp, which is defined as

$$
f_{c}(t)= \begin{cases}0 ; & 0 \leq t<\frac{1}{4} \\ t ; & \frac{1}{4} \leq t \leq \frac{3}{4} \\ 0 ; & \frac{3}{4}<t \leq 1\end{cases}
$$

and $\sigma=0.20$ for the chirp

$$
f_{c}(t)= \begin{cases}0 ; & 0 \leq t<\frac{1}{4} \\ \sin \left(2 \pi t+20 \pi t^{2}\right) ; & \frac{1}{4} \leq t \leq \frac{3}{4} \\ 0 ; & \frac{3}{4}<t \leq 1 .\end{cases}
$$

Fig. 2(a) shows 500 samples of noisy data corresponding to the ramp, which are generated by using the routine g05ddf of

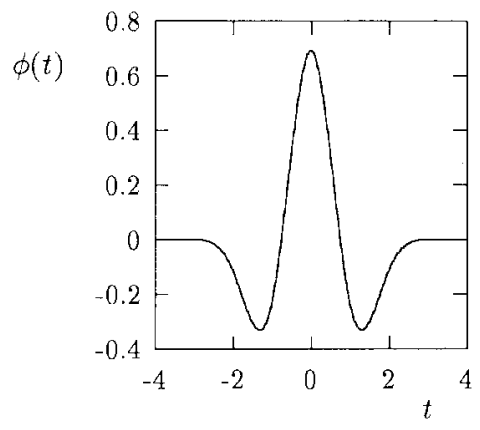

(a)

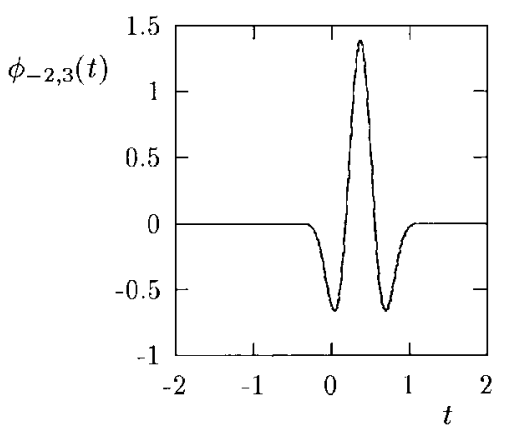

(b)

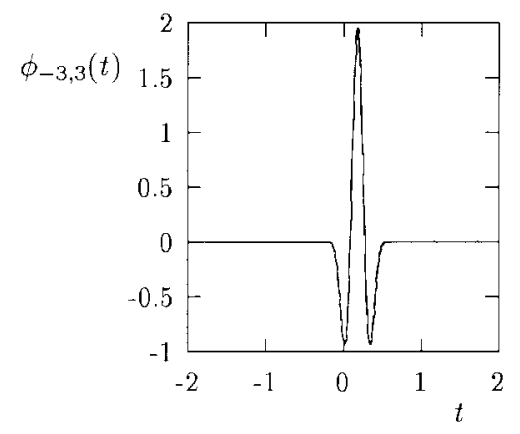

(c)

Fig. 1. (a) Mother wavelet $\phi(t)$. (b) Wavelet $\phi_{m, n}(t)$ for $m=-2, n=3$. (c) Wavelet $\phi_{m, n}(t)$ for $m=-3, n=3$.

the NAG FORTRAN library [9]. The thicker line of Fig. 2(b) represents the original clean signal for comparison, and the thinner line represents the reconstruction obtained through both the wavelet and orthonormal representation for TOL $=$ $1 e-12$ [cf. (52)]. The value $m_{1}$ was found to be equal to -2 .

Fig. 3 shows the coefficients of the wavelet representation. Although only a few coefficients are significant, they are very large numbers. In this case, the spectrum of the operator $\hat{F}_{M N}^{*}$ has an obvious cut, and therefore, the decision as to which of the singular values should be considered zero is not very sensitive to the TOL number in (52). The same number $r=13$ of singular values to be considered nonzero is obtained for values of TOL that range from $1 e-9$ to $1 e-12$. If $r$ is decreased to be 12 or 11 , the coefficients are still large numbers, but the approximation of the signal becomes much poorer. A set of coefficients of small size is obtained for $r=10$ (see Fig. 4); however, as is shown in Fig. 5, the approximation, which is obtained by this set of coefficients, is not a satisfactory representation of the original signal. On 


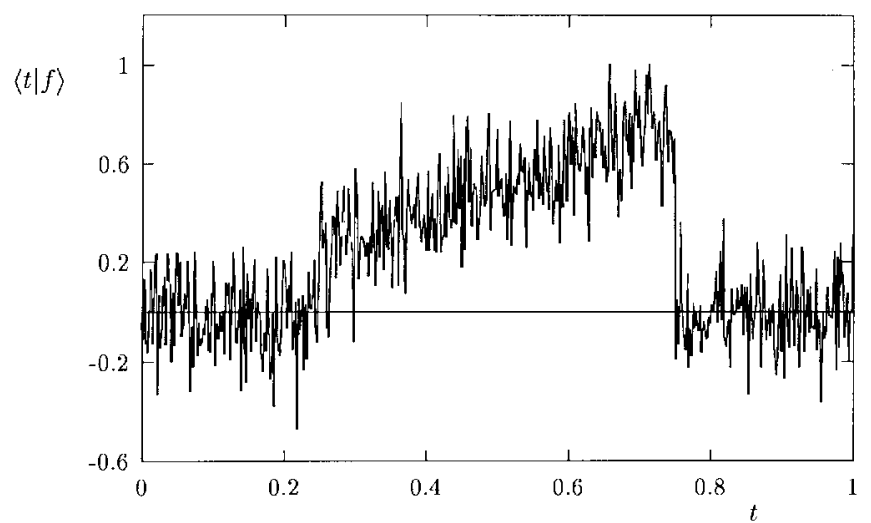

(a)

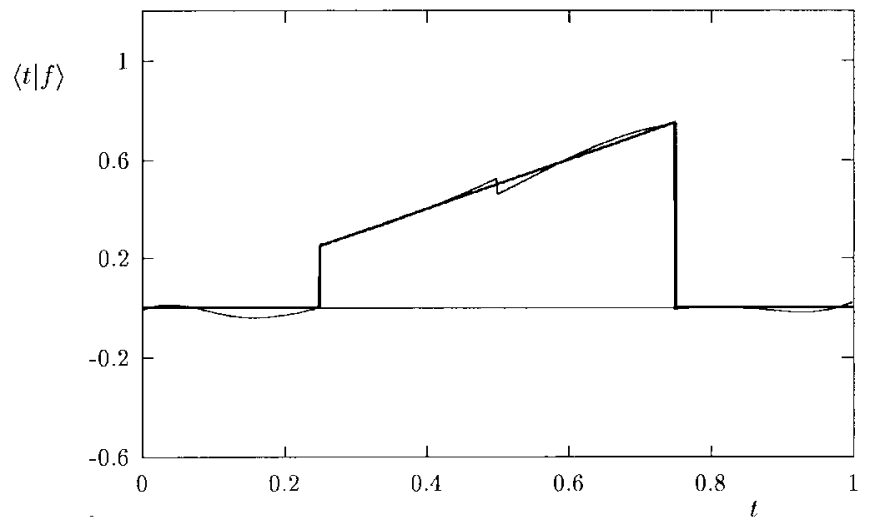

(b)

Fig. 2. (a) Input noisy data corresponding to the ramp example and (b) thicker line plots the original signal. The thinner line plots the reconstruction obtained through both the wavelet and orthonormal representations.

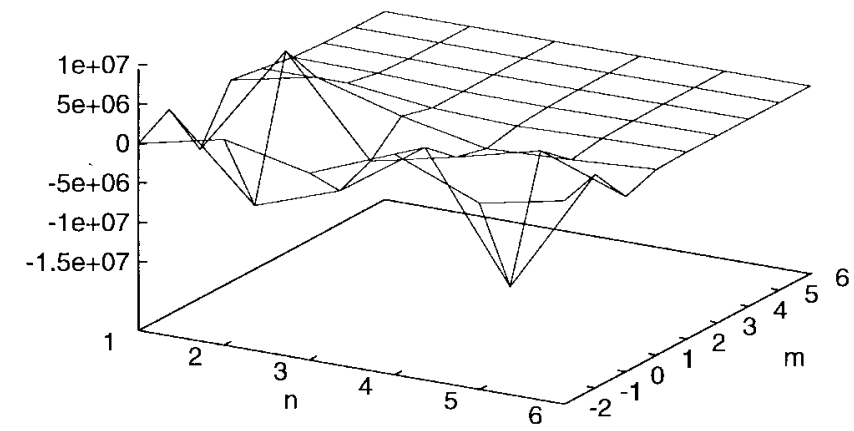

Fig. 3. Vertical axis shows the wavelet representation coefficients $c_{m, n}=\left\langle\tilde{\phi}_{m, n} \mid f\right\rangle$ corresponding to the ramp example.

the other hand, the circles in Fig. 6 correspond to the values of the coefficients of the orthonormal expansion, and this is clearly a more economical representation. Moreover, it can be seen that those coefficients for $l=11,12$, and 13 have significant value, and therefore, the corresponding singular values should be considered nonzero in order to achieve a good approximation of the signal.

Fig. 7(a) shows the noisy data corresponding to the chirp pulse generated by using the same routine as in the previous case. In this case, we deal with 1000 samples. In Fig. 7(b), the thicker line corresponds to the original clean signal for comparison, whereas thinner line indicates the result obtained

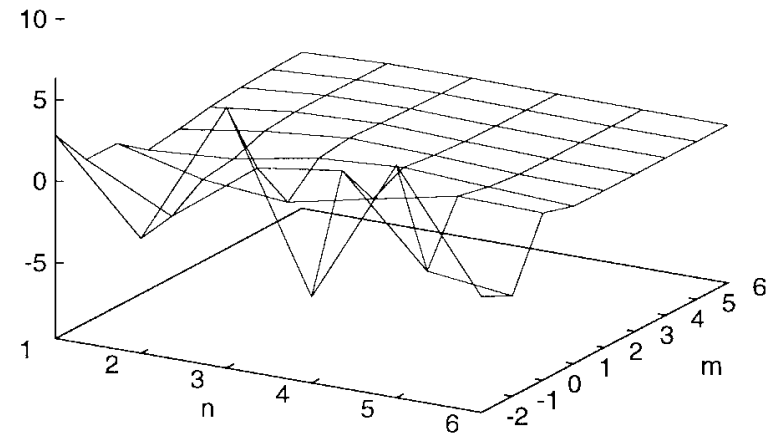

Fig. 4. Vertical axis showing the wavelet representation coefficients $c_{m, n}=\left\langle\tilde{\phi}_{m, n} \mid f\right\rangle$ obtained by cutting the singular values at $r=10$ in order to regularize the solution.

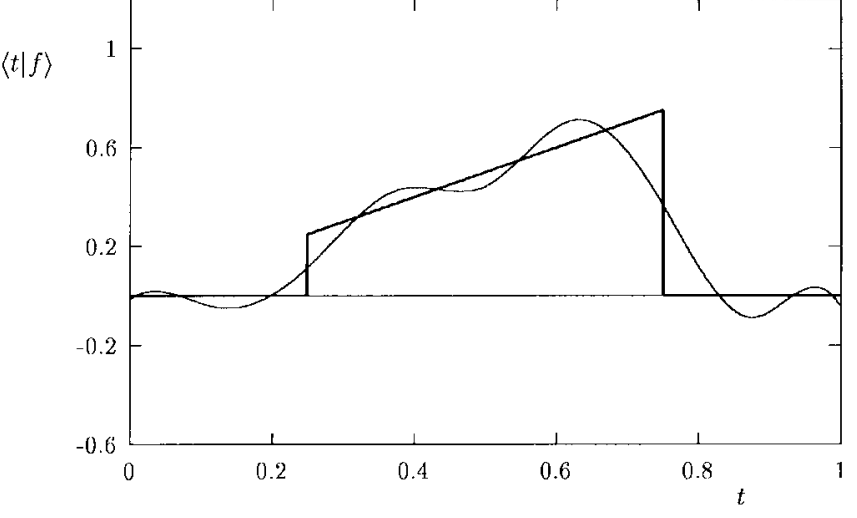

Fig. 5. Thicker line plots the original signal. The thinner line plots the approximation obtained if the singular values are cut in order to obtain the set of regularized coefficients of Fig 4.

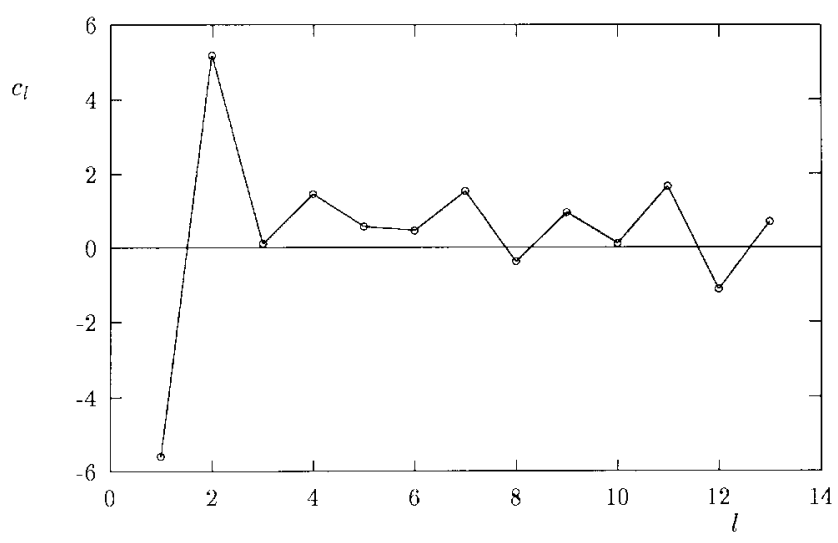

Fig. 6. Orthogonal representation coefficients $c_{l}=\left\langle\bar{\varphi}_{l} \mid f\right\rangle$ corresponding to the ramp example.

through the present approach for TOL $=1 e-12$. After five iterations, $m_{1}$ is fixed to -5 . Fig. 8 shows the coefficients for the wavelet decomposition, and they clearly have the same feature as above, namely, that they are very large numbers. The coefficients of the orthonormal representation are shown in Fig. 9. As can be seen in that figure, in this case, coefficients corresponding to $l>48$ are all of very small size in comparison with the most significant ones. Thus, singular 


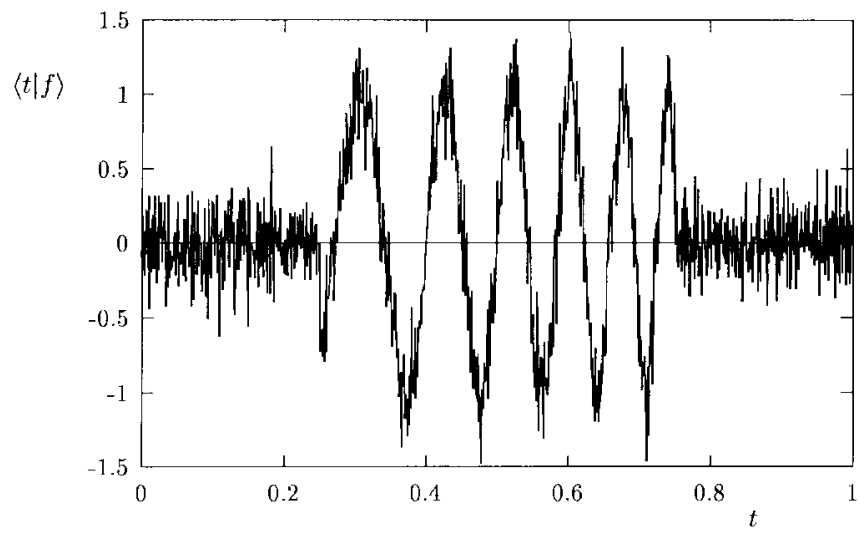

(a)

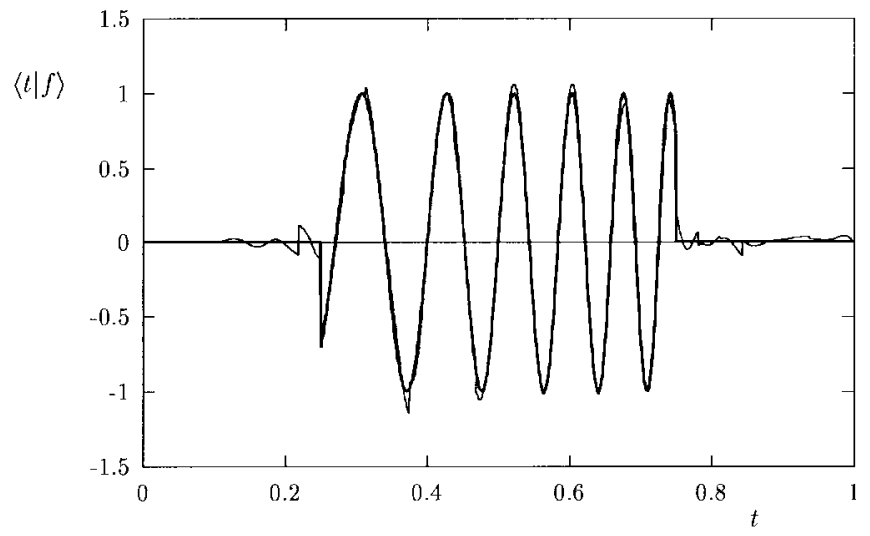

(b)

Fig. 7. (a) Input data corresponding to the chirp example. (b) Same details as in Fig. 2(b).

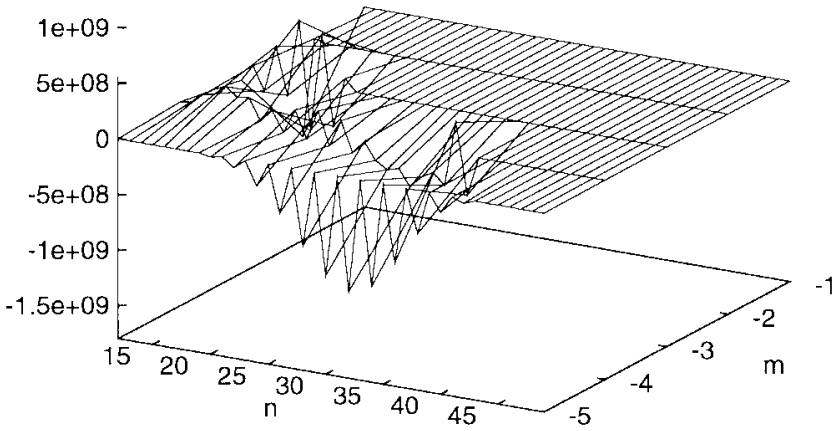

Fig. 8. Same details as in Fig. 3 for the chirp example.

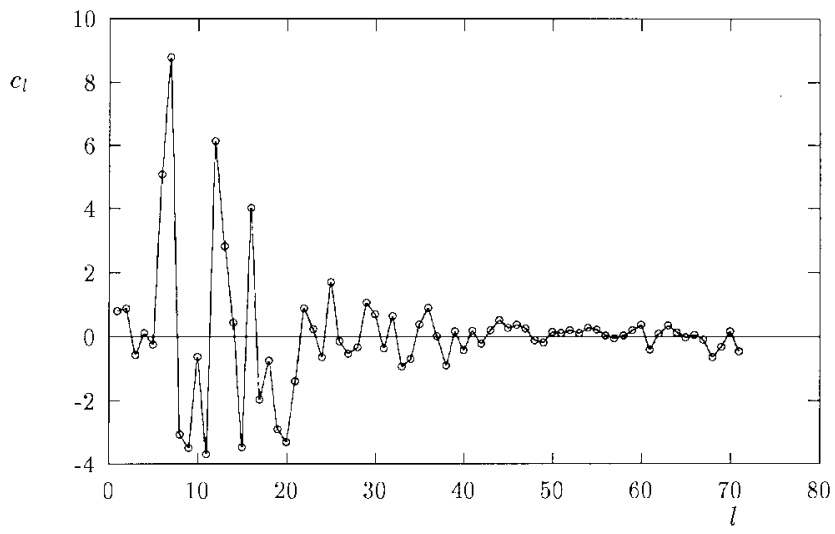

Fig. 9. Same details as in Fig. 6 for the chirp example.

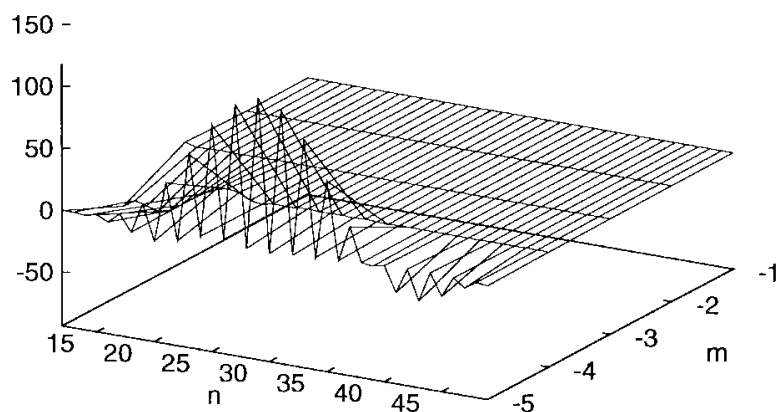

Fig. 10. Vertical axis showing the wavelet representation coefficients $c_{m, n}=\left\langle\tilde{\phi}_{m, n} \mid f\right\rangle$ obtained by cutting the singular values at $r=48$ in order to regularize the solution.

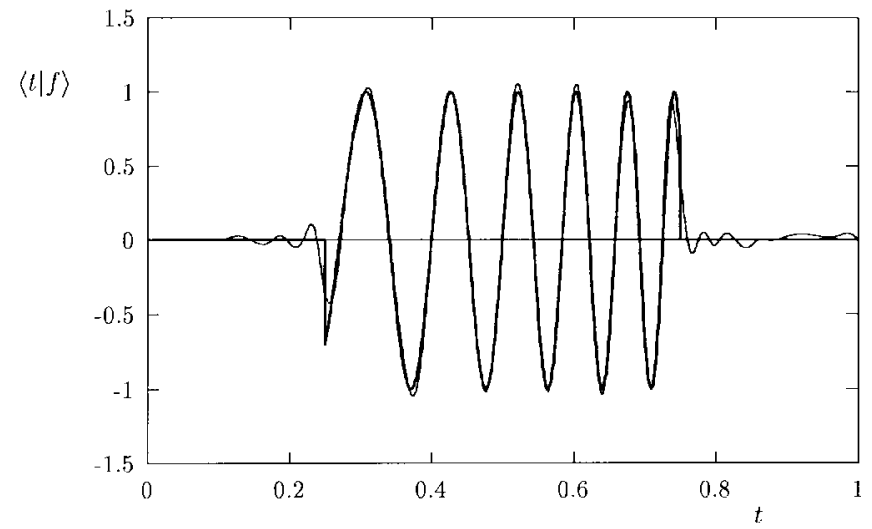

Fig. 11. Thicker line plots the original signal. The thinner line plots the approximation obtained if the singular values are cut in order to obtain the set of regularized coefficients of Fig. 10.

values corresponding to $r>48$ may be cut without affecting resolution in the representation of the signal. In Fig. 10, it is shown that by cutting the singular values at $r=48$, a "regularized" solution in terms of wavelets is obtained. The signal approximation that is obtained through this set of coefficients is shown in Fig 11. It is seen then that the orthogonal representation we propose here is also a tool to tackle the usually difficult problem of deciding which singular values should be considered to be zero.

In the next example, the time domain was expanded to [0,3], and 1500 samples were taken. The signal to be reconstructed is composed of the following sinusoids on multilevel echelon:

$$
f_{c}(t)= \begin{cases}\sin (16 \pi t) ; & 0 \leq t<1 \\ 3+\sin (14 \pi t) ; & 1 \leq t<2 \\ 6+\sin (12 \pi t) ; & 2 \leq t<3\end{cases}
$$

In this case, we consider the variance of the noise changes with time as

$$
\sigma= \begin{cases}\sigma_{1}=0.2 ; & 0 \leq t<1 \\ \sigma_{2}=0.4 ; & 1 \leq t<2 \\ \sigma_{3}=0.7 ; & 2 \leq t<3\end{cases}
$$


Condition (60) is satisfied by $m_{1}=-4$ as this is the maximum $m_{1}$ value for which we have

$$
\begin{aligned}
& \frac{1}{500}\left[\chi_{[0,1]} \sum_{i=1}^{500}\left(\left\langle t_{i} \mid f\right\rangle-\left\langle t_{i} \mid f^{*}\right\rangle\right)^{2}\right. \\
& +\chi_{[1,2]} \sum_{i=501}^{1000}\left(\left\langle t_{i} \mid f\right\rangle-\left\langle t_{i} \mid f^{*}\right\rangle\right)^{2} \\
& \left.\quad+\chi_{[2,3]} \sum_{i=1001}^{1500}\left(\left\langle t_{i} \mid f\right\rangle-\left\langle t_{i} \mid f^{*}\right\rangle\right)^{2}\right] \\
& \leq \chi_{[0,1]} \sigma_{1}^{2}+\chi_{[1,2]} \sigma_{2}^{2}+\chi_{[2,3]} \sigma_{3}^{2}
\end{aligned}
$$

where $\chi_{\left[t_{1}, t_{2}\right]}$ is the characteristic function of the $\left[t_{1}, t_{2}\right]$ interval, which is defined as

$$
\chi_{\left[t_{1}, t_{2}\right]}= \begin{cases}1 ; & t_{1} \leq t \leq t_{2} \\ 0 ; & \text { otherwise }\end{cases}
$$

The noisy data and the achieved reconstruction for TOL $=$ $1 e-12$ are shown in Fig. 12(a) and (b), respectively. The coefficients corresponding to the wavelet and orthonormal representations are plotted in Figs. 13 and 14. Similar comments can be made as in the previous example. If the cut in the spectrum of singular values is decided by setting $r=61$ taking into account that for $l>61$ the orthogonal coefficients are all of small size, the regularized solution, which is shown in Fig. 15, is obtained. It is important to remark that to truncate the singular values at $r=61$ corresponds to setting TOL $=$ $1 e-4$. This value of TOL is very much larger than the error in the calculus; however, setting the value of TOL $=1 e-4$, based on the analysis of the orthogonal coefficients, gives rise to a regularized solution. The thin line in Fig. 16 shows the approximation obtained by such a regularized solution, and as can be seen, this is a slightly worse approximation than the one obtained by using the whole spectrum of nonzero singular values.

\section{CONCLUSIONS}

A mathematical scheme for data compression and noise reduction has been presented. As a starting point, the use of well-localized wavelets is proposed in order to generate a representation subspace capable of reproducing the original signal and excluding additive noise.

Our proposal is different from the normal frame approach in that we do not use the dual frames in the Hilbert space to construct the approximation of the signal in the working subspace. Instead, we calculate the dual vectors in such a subspace. We warn about the implications of the last procedure. The concomitant inverse problem has been addressed, and a solution, in the sense of a minimum norm, was found. The causes and effects of the undesired behavior of such a solution have been examined.

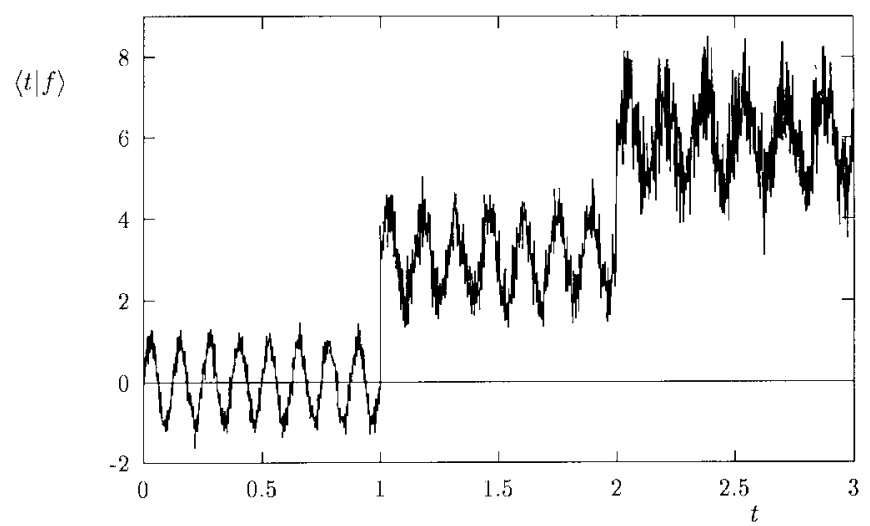

(a)

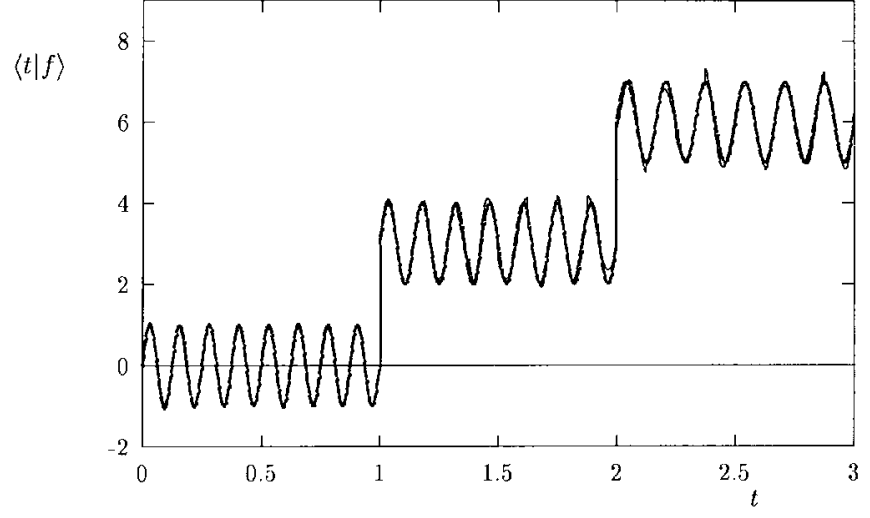

(b)

Fig. 12. (a) Input data corresponding to three sinusoids on multilevel echelon. (b) Same details as in Fig. 2(b).

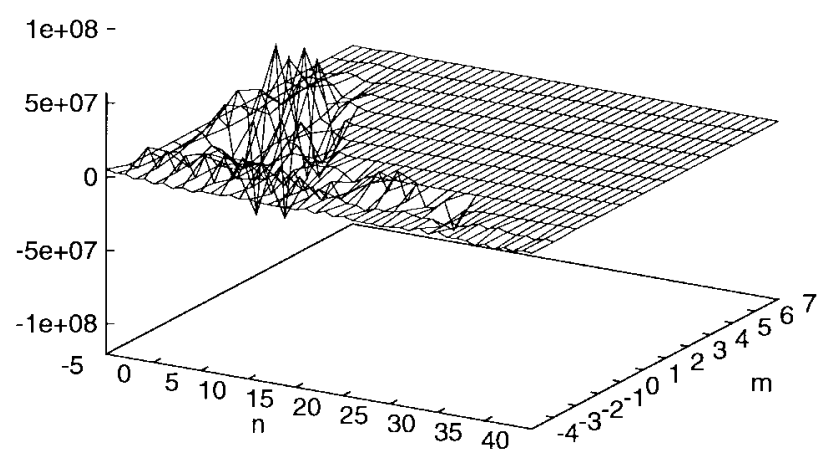

Fig. 13. Same details as in Fig. 3 for the case of three sinusoids on multilevel echelon.

We are led to conclude that there exists an ill-condition aspect inherent in the inverse problem that arises when one calculates the dual vectors in a subspace spanned by a finite number of frame elements in $L^{2}[0, T]$. The representation in terms of such a vectors gives rise to solutions with a very large norm. An alternative orthonormal representation for the generated subspace has been proposed in this paper that conserves the signal norm and that, therefore, overcomes the ill-conditioning problem. Both the orthonormal and wavelet representations have been shown to yield identical approxima- 


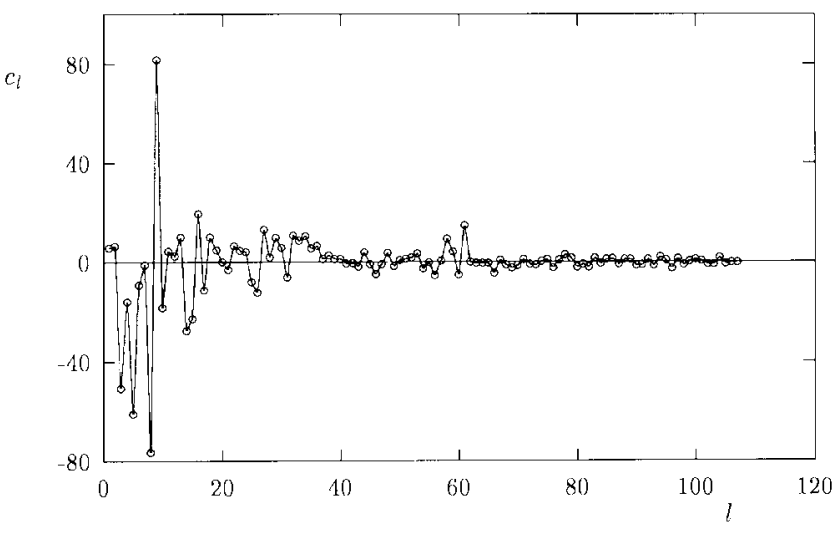

Fig. 14. Same details as in Fig. 6 for the case of three sinusoids on multilevel echelon.

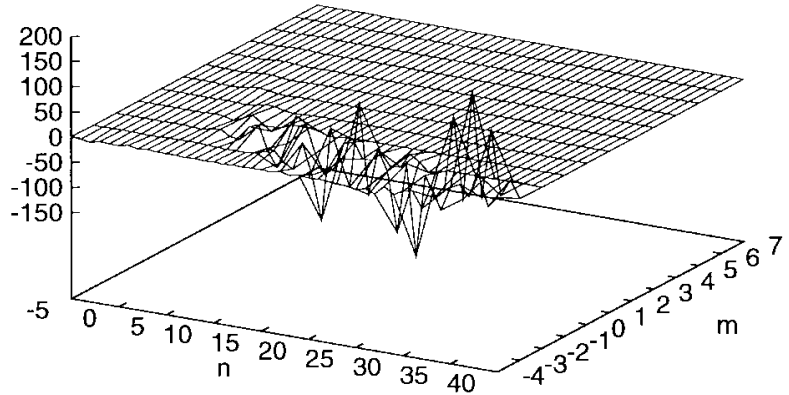

Fig. 15. Vertical axis showing the wavelet representation coefficients $c_{m, n}=\left\langle\tilde{\phi}_{m, n} \mid f\right\rangle$ obtained by cutting the singular values at $r=61$ in order to regularize the solution.

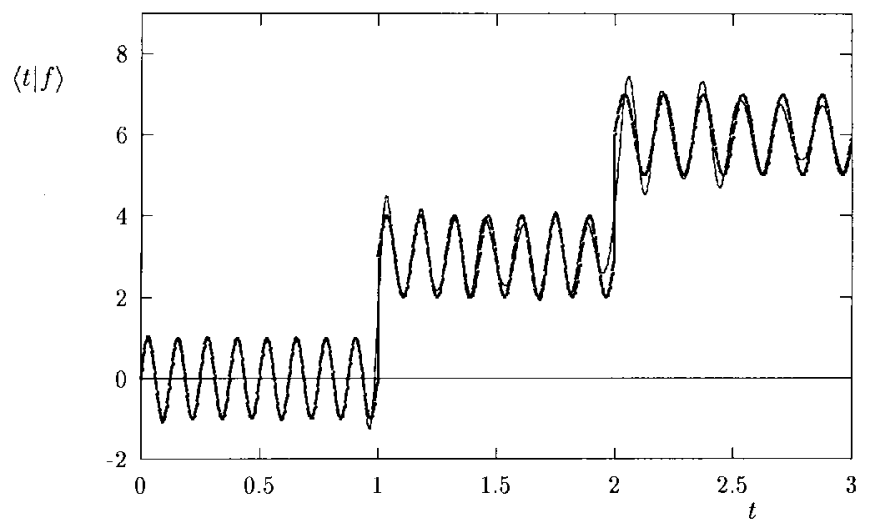

Fig. 16. Thicker line plots the original signal. The thinner line plots the approximation obtained if the singular values are cut in order to obtain the set of regularized coefficients of Fig. 15.

tions for signals outside the working subspace. However, the new approach has features that are superior to the calculation of the duals vectors in the working subspace, particularly in eliminating redundancy and in preserving the signal norm. Moreover, the orthogonal approach also provides a tool for regularizing the coefficients of the wavelet representations by truncating the spectrum of singular values in those cases in which this can be done; it also indicates when this is not possible (Example 1).

A procedure to reduce the effect of zero mean Gaussian noise has been examined. As expected, the reconstruction of the original signals improves as the variance of the noise decreases. The discontinuity that appears in the ramp example, which is not present in the original signal, disappears when the noise standard deviation is reduced to $5 \%$ of the peak uncontaminated signal.

\section{ACKNOWLEDGMENT}

The authors wish to thank an unknown referee whose comments improved this paper considerably. L. Rebollo-Neira wishes to express her gratitude to Dr. T. J. Seller for his help and assistance during her stay in England.

\section{REFERENCES}

[1] P. A. M. Dirac, The Principles of Quantum Mechanics. Oxford, U.K.: Oxford Univ. Press, 1958

[2] I. Daubechies, A. Grossmann, and Y. Meyer, "Painless nonorthogonal expansions," J. Math. Phys., vol. 27, pp. 1271-1283, 1986.

[3] I. Daubechies, "Orthogonal bases of compactly supported wavelets," Commun. Pure Appl. Math., vol. 41, pp. 909-996, 1988.

[4] _ "The wavelets transform, time frequency localization and signal analysis," IEEE Trans. Inform. Theory, vol. 36, pp. 961-1005, 1990.

[5] _ Ten Lectures on Wavelets. Philadelphia, PA: Soc. Indus. Applied Math., 1992.

[6] R. J. Duffin and A. C. Shaffer, "A class of nonharmonic Fourier series," Trans. Amer. Math. Soc., vol. 72, pp. 341-366, 1952.

[7] C. Heil and D. Walnut, "Continuous and discrete wavelet transforms" SIAM Rev., vol. 31, pp. 628-666, 1989.

[8] S. Mallat, "A theory for multiresolution signal decomposition: The wavelet representation," IEEE Trans. Patt. Anal. Machine Intell., vol. 11, pp. 674-693, 1989.

[9] NAG Fortran Library Manual, Numerical Algorithms Group, 1987.

[10] Y. Meyer, Wavelets and Operators. Cambridge, U.K.: Cambridge Univ. Press, 1992.

[11] M. Reed and B. Simon, Functional Analysis. New York: Academic, 1980.

[12] J. H. Wilkinson, "Singular-values decomposition-Basic aspects," in Numerical Software-Needs and Availability. London, U.K.: Academic, 1978.

[13] R. M. Young, An Introduction to Nonharmonic Fourier Series. New York: Academic, 1980

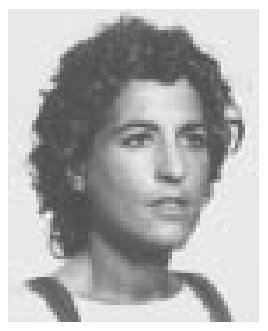

Laura Rebollo-Neira received the Ph.D. degree in physics from La Plata University, La Plata, Argentina, in 1986.

Since 1987, she has been with the Department of Physics, La Plata University, as a Member of the Scientific Staff of the Scientific Commission of the Province of Buenos Aires, Argentina. Between 1992 and 1995, she was an Academic Visitor at the Department of Electrical and Electronic Engineering, Imperial College, London, U.K. She has worked in physical modeling and maximumentropy-based inversion methods. Her current research interests include wavelets, frames, mathematical methods, and signal processing theory.

Dr. Rebollo-Neira is a Fellow of the Comisión de Investigaciones Científicas de la Provincia de Buenos Aires (CICPBA). 


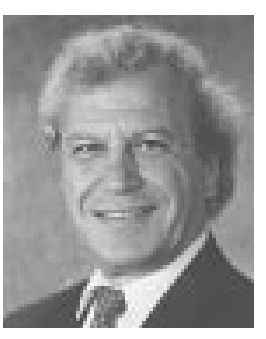

Anthony G. Constantinides (SM'78) is a Professor of Signal Processing and the Head of the Signal Processing and Digital Systems Section of the Department of Electrical Engineering, Imperial College, London, U.K.. He has been actively involved with research in various aspects of digital filter design, digital signal processing, and communications for a number of years. His research spans a wide range of digital signal processing both from the theoretical as well as the practical points of view. His recent work has been directed toward the demanding signal processing problems arising from the area of telecommunications. He has published a range of books and papers in learned journals in the area of digital signal processing and its applications.

Prof. Constantinides served as the first President of the European Association for Signal Processing (EURASIP) and has contributed in this capacity to the establishment of the European Journal for Signal Processing. He has been, and is currently serving as, a member of many technical program committees of IEEE International Conferences. He organized the first ever international series of meetings on digital signal processing in London in 1967 and in Florence, Italy (with V. Cappellini). In 1985, he was awarded the Honor of Chevalier, Palmes Academiques, by the French Government, and in 1996, he was promoted to Officer, Palmes Academiques. He holds several visiting professorships and other fellowships and honors around the world. He presently is serving as a member of the Signal Processing Society Technical Committee on Neural Networks for Signal Processing of the IEEE Signal Processing Society.

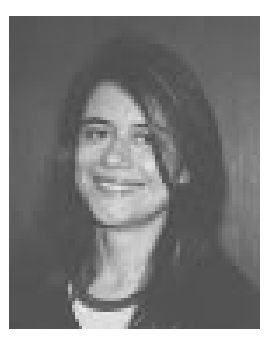

Tania Stathaki was born in Athens, Greece. She received the M.Eng. degree in Electronics and Computer Engineering from the National Technical University of Athens in 1991 and the Ph.D. degree in signal processing from Imperial College, London, U.K., in 1994.

Since 1994, she has been a Research Associate with the Department of Electrical and Electronic Engineering, Imperial College. Her current research interests are in the areas of nonlinear systems, image processing, and higher order statistics. 